\title{
Beyond Shapes: Lists with Ordered Data
}

\author{
Kshitij Bansal $^{1, \star}$, Rémi Brochenin ${ }^{2, \star \star}$, and Etienne Lozes $^{2}$ \\ 1 Chennai Mathematical Institute \\ kshitij@cmi.ac.in \\ 2 LSV, ENS Cachan, CNRS \\ \{brocheni, lozes\}@lsv.ens-cachan.fr
}

\begin{abstract}
Standard analysis on recursive data structures restrict their attention to shape properties (for instance, a program that manipulates a list returns a list), excluding properties that deal with the actual content of these structures. For instance, these analysis would not establish that the result of merging two ordered lists is an ordered list. Separation logic, one of the prominent framework for these kind of analysis, proposed a heap model that could represent data, but, to our knowledge, no predicate dealing with data has ever been integrated to the logic while preserving decidability. We establish decidability for (first-order) separation logic with a predicate that allows to compare two successive data in a list. We then consider the extension where two data in arbitrary positions may be compared, and establish the undecidability in general. We define a guarded fragment that turns out to be both decidable and sufficiently expressive to prove the preservation of the loop invariant of a standard program merging ordered lists. We finally consider the extension with the magic-wand and prove that, by constrast with the data-free case, even a very restricted use of the magic wand already introduces undecidability.
\end{abstract}

\section{Introduction}

Data-ordering and shape analysis. Providing automatic methods for faults detection in programs manipulating recursive mutable data structures is a longstanding problem. Shape analysis is a well established approach that may detect faults due to in-depth properties of the heap, like creating a cycle in an acyclic list. Prominent logics that integrate such an analysis are separation logic [1, pointer assertion logic PAL 99, TVLA [10], LRP (logic of reachable patterns) [16], or alias logic [4, to quote a few examples. A common feature in these analyses is that they completely forget the data held in the recursive structures, focusing on the shape of the structure. As a consequence, ordering properties are out of the scope of these analyses: for instance, one cannot check or even specify that the reverse of a sorted list is a sorted list. Extensions of shape analysis have been proposed for ordering properties, stability properties,

\footnotetext{
* Supported by P2R MODISTE-COVER/Timed-DISCOVERI, a project under the Indo-French Networking Programme.

** Supported by a DGA/CNRS fellowship.
}

L. de Alfaro (Ed.): FOSSACS 2009, LNCS 5504, pp. 425 439 2009.

(C) Springer-Verlag Berlin Heidelberg 2009 
and size properties, in shape graphs [3], in the TVLA approach [11], and in the separation logic approach [12] to cite a few. This paper studies the rather more theoretical issue of the decidability of the satisfiability problem. It proposes a general approach for reducing the shapes handling ordering properties to pure shapes, and stress some natural limitations we should put on the data properties we would like to check automatically.

Data-ordering in separation logic. Our approach lies in the framework of separation logic [14. In essence, separation logic extends first order logic with two substructural connectives: the separation connective $(*)$ and its adjoint (the separating implication $*$, also known as the magic wand). These connectives are convenient to express pre and post conditions of all standard heap-manipulating instructions. For instance, the strongest post condition $\operatorname{Post}(\mathrm{x}:=$ new, $A)$ of a memory allocation instruction can be expressed by $\mathbf{x} \mapsto-* \exists x . A\{x / \mathbf{x}\}$. This formula involves two more ingredients : the use of first-order logical variables, that here quantify over the memory location of $\mathbf{x}$ before allocation, and the points-to predicate $\hookrightarrow$. (or its precise version in this example). We extend the logic with the predicate $\operatorname{val}(x) \leq \operatorname{val}(y)$ that asserts that the value stored at the location $x$ is smaller than the one stored at $y$, which in particular allows to define the predicate

$$
x \stackrel{\leq}{\rightrightarrows} \stackrel{\text { def }}{\equiv} x \hookrightarrow y \wedge \operatorname{val}(x) \leq \operatorname{val}(y)
$$

and $x \stackrel{\geq}{\longleftrightarrow} y$ accordingly. We call these predicates short-distance comparisons, and by contrast $\operatorname{val}(x) \leq \operatorname{val}(y)$ is called long-distance comparison. We moreover say that such a long-distance comparison is guarded if $x$ or $y$ is an open variable.

Separation logic's decidability. The decidability of the satisfiability problem for separation logic has been intensively studied so far: first-order separation logic over heap models with at least two selectors (record fields) is known to be undecidable [7] by containment of finite satisfiability for classical predicate logic with one binary relation [15] (even with no separating connectives). On the other hand, first-order separation logic over heaps with one selector has been proved to be decidable when the magic-wand is dropped [6], by reduction to monadic second order logic over functional graphs, but becomes undecidable in presence of magic wand. To our knowledge, nothing was known about first-order separation logic with data. The following table summarizes the results we present in this paper:

\begin{tabular}{c|c} 
Undecidable & $\begin{array}{c}\text { long distance comparison without } * \\
\text { short distance comparison with }(\text { restricted) } *\end{array}$ \\
\hline Decidable & short + guarded long distance comparisons without $\rightarrow$
\end{tabular}

The decidability result comes from a reduction to monadic second order logic over functional graphs. The translation is strongly inspired by the one for separation logic over lists without data [6], but involves some non-trivial complications for ensuring the coherence of data abstraction. The undecidability results are obtained by reduction to first-order logic over (finite) data words, which was proved undecidable 28$]$. 
Case study. In order to illustrate the practical relevance of our results, we consider a very standard merge-sort program. Checking that any formula is a correct loop invariant requires in general to deal with the magic-wand connective, which leads to our undecidable fragments. However, for the loop invariant one may think about (that is, all working lists are ordered lists) the magic wand can be eliminated, and the formula considered falls into the decidable fragment.

Outline of the paper. Section 2 introduces our separation logic over lists with data. In Section 3. we illustrate on the merge program how the logic can deal with relevant loop invariants. In Section [4 we establish the decidability of the short distance comparison. Section [5] deals with the case of guarded and nonguarded long-distance comparison, whereas Section 6 explains the undecidability of the logic in the presence of the magic wand.

\section{Preliminaries}

In this section, we introduce first the separation logic with data considered in this work, then the monadic second order logic to which we reduce to. These logics are based on different classes of models: our separation logic deals with lists with data, whereas the monadic second order logic deals with shapes, e.g. lists without data.

\subsection{A Separation Logic for Lists with Ordered Data}

Memory model. We assume an infinite, totally ordered set $($ Dat,$\leq)$ of data, and range over a particular datum with $\alpha, \beta$. We moreover assume an infinite set Loc of locations, ranged over with $l, l^{\prime}$ etc. and an infinite set Var of variables, ranged over with either $x, y, z$ or $\mathbf{x}, \mathbf{y}, \mathbf{z}$ etc. Variables can be interpreted as both variables from the programs or logical variables quantifying over locations; the main difference between both is that program variables are never quantified in the formula. We safely identify them and will use the font convention $\mathbf{x}, \mathbf{y}$ to emphasize that a variable should be understood as a program variable. In the latter, we may use the standard notation $A\{y / x\}$ for the formula $A$ in which $x$ replaces $y$.

Following the standard semantics of separation logic, we define a memory state as a pair of a store $s$ and a heap $h$ such that:

$-s:$ Var $\rightarrow$ Loc

$-h:$ Loc $\rightarrow($ Loc $\times$ Dat $)$ is a partial function with finite domain.

We write $\operatorname{dom}(h)$ to denote the domain of $h$ and $\operatorname{ran}(h)$ to denote its range. For $\mathcal{Z} \subseteq \operatorname{dom}(h)$, We write $h_{\mid \mathcal{Z}}$ to denote the restriction of $h$ to $\mathcal{Z}$. We write fst and snd to denote the first and second projection on a product set. We write $h \perp h^{\prime}$ if $\operatorname{dom}(h) \cap \operatorname{dom}\left(h^{\prime}\right)=\emptyset$, and the heap composition $h * h^{\prime}$ is defined as $h \cup h^{\prime}$ when $h \perp h^{\prime}$. 
Example 1 (Ordered lists). Programs manipulating ordered lists of integers can be modeled choosing Dat $=\mathbb{Z}$ with the standard order. The same holds for lists of reals, lists of naturals, and so on.

Example 2 (Fine-grained concurrent lists). Dat could be thought as the state of a lock at the current node, that is the identifier of the thread holding the node (or some constant for an available lock). Here, the ordering on data is not relevant, but the equality between data is. For such a model, one may want to express, for instance, that every thread holds the locks of at most two nodes of a list, and that these nodes are necessarily consecutive.

Separation logic. We now define our assertion language $\mathrm{SL}_{<}$by extending the standard separation logic with a comparison predicate. We assume a set DVar of data variables, ranged over with $v, w$, etc. A valuation interpreting data variables is a function $\rho:$ DVar $\rightarrow$ Dat.

Formulae of $\mathrm{SL}_{<}$are defined by the grammar below.

$$
\phi::=\neg \phi|\phi \wedge \phi| \exists x . \phi|\exists v . \phi| x \hookrightarrow y|\operatorname{val}(x) \leq v| \operatorname{val}(x) \geq v|x=y| \phi * \phi \mid \phi \rightarrow \phi
$$

The semantics of the formulae is defined as usual, with the expected definition for the predicates $\operatorname{val}(x) \leq v$ and $\operatorname{val}(x) \geq v$.

$$
\begin{aligned}
& (s, h), \rho=_{\mathrm{SL}} \phi \wedge \psi \quad \text { iff } \quad(s, h), \rho=_{\mathrm{SL}} \phi \text { and }(s, h), \rho \models_{\mathrm{SL}} \psi \\
& (s, h), \rho \models_{\mathrm{SL}} \neg \phi \quad \text { iff } \operatorname{not}(s, h), \rho \models_{\mathrm{SL}} \phi \\
& (s, h), \rho \models_{\mathrm{SL}} \exists x . \phi \quad \text { iff there is } l \in \text { Loc such that }(s[x \mapsto l], h), \rho \models_{\mathrm{SL}} \phi \\
& (s, h), \rho \models_{\mathrm{SL}} \exists v . \phi \quad \text { iff there is } \alpha \in \text { Dat such that }(s, h), \rho[v \mapsto \alpha] \models_{\mathrm{SL}} \phi \\
& (s, h), \rho \models_{\mathrm{SL}} x \hookrightarrow y \quad \text { iff there is } \alpha \in \text { Dat such that } h(s(x))=(s(y), \alpha) \\
& (s, h), \rho \models_{\mathrm{SL}} \operatorname{val}(x) \leq v \text { iff there is } \alpha \in \text { Dat and } l \in \text { Loc such that } \\
& h(s(x))=(l, \alpha), \text { and } \alpha \leq \rho(v) \\
& (s, h), \rho=_{\mathrm{SL}} \operatorname{val}(x) \geq v \text { iff there is } \alpha \in \text { Dat and } l \in \text { Loc such that } \\
& h(s(x))=(l, \alpha), \text { and } \alpha \geq \rho(v) \\
& (s, h), \rho=_{\mathrm{SL}} x=y \quad \text { iff } \quad s(x)=s(y) \\
& (s, h), \rho \models_{\mathrm{SL}} \phi_{1} * \phi_{2} \quad \text { iff } \quad \text { there are two heaps } h_{1}, h_{2} \text { such that } \\
& h=h_{1} * h_{2} \text { and }\left(s, h_{i}\right), \rho=_{\mathrm{SL}} \phi_{i}, i=1,2 \\
& (s, h), \rho \models_{\mathrm{SL}} \phi_{1} * \phi_{2} \quad \text { iff } \quad \text { for all } h^{\prime} \text {, if } h \perp h^{\prime} \text { and }\left(s, h^{\prime}\right), \rho=_{\mathrm{SL}} \phi_{1} \text {, } \\
& \text { then }\left(s, h * h^{\prime}\right), \rho=_{\mathrm{SL}} \phi_{2}
\end{aligned}
$$

Note that, due to our memory model, the natural semantics of $\operatorname{val}(x) \leq v$ implies in particular $\exists z . x \hookrightarrow z$.

Derived formulae. We use standard notations $\vee, \forall, \Rightarrow$, and write $\operatorname{val}(x)=v$, $\operatorname{val}(x) \leq \operatorname{val}(y), \ldots$ for the obvious combinations of comparison predicates. We write precisely $(A)$ to denote $A \wedge \neg(A * \exists x, y . x \hookrightarrow y)$. We also abbreviate $\phi \exists \psi$ for the sometimes called septraction connective defined by $\neg(\phi \rightarrow \neg \neg \psi)$. We use the wildcard notation, e.g. $x \hookrightarrow-$ for $\exists y . x \hookrightarrow y$, the so-called precise predicates $\mapsto$ 
(e.g. $x \mapsto y$ abbreviates precisely $(x \hookrightarrow y))$, and equality over vectors $\left(x_{1}, . ., x_{n}\right)=$ $\left(y_{1}, . ., y_{n}\right)$. We will also use the following shorthands:

$$
\begin{aligned}
& x \stackrel{\leq}{\hookrightarrow} y \quad \text { for } x \hookrightarrow y \wedge \operatorname{val}(x) \leq \operatorname{val}(y) \text {, and } x \stackrel{\geq}{\hookrightarrow} y \text { accordingly, } \\
& x \mapsto(y, v) \text { for } x \mapsto y \wedge \operatorname{val}(x)=v \\
& x{ }^{*} y \quad \text { for } x=y \vee(\top *((x \hookrightarrow-) \wedge(-\hookrightarrow y) \wedge \neg(-\hookrightarrow x) \wedge \neg(y \hookrightarrow-) \\
& \wedge \forall z \notin\{x, y\} .((z \hookrightarrow-) \Leftrightarrow(-\hookrightarrow z)))) \\
& x \hookrightarrow^{+} y \quad \text { for } \exists z \cdot x \hookrightarrow z \wedge z \hookrightarrow^{*} y, \\
& \operatorname{decls}(x, y) \text { for } \operatorname{precisely}(x=y) \quad \vee \quad x \mapsto y \\
& \vee \text { precisely }\left(\exists y^{\prime} . x \hookrightarrow^{+} y^{\prime} \wedge y^{\prime} \hookrightarrow y \wedge \forall z \cdot\left(z \hookrightarrow^{+} y^{\prime}\right) \Rightarrow(z \stackrel{\geq}{\hookrightarrow}-)\right)
\end{aligned}
$$

We christen the $\stackrel{\leq}{\leftrightarrows}$ predicate short-distance comparison, and by contrast refer to $\operatorname{val}(x) \leq \operatorname{val}(y)$ as long-distance comparison. The binary predicate $x \hookrightarrow{ }^{*} y$ is the accessibility relation (see [6]); it asserts that $(\text { fst } \circ h)^{n}(s(x))=s(y)$ for some $n \geq 0$.

The binary predicate decls $(x, y)$ characterises a heap composed of a single list segment with data sorted in the decreasing order.

\subsection{A Monadic Second Order Logic over Memory Shapes}

Memory shapes. We define memory shapes as the abstraction of a memory heap forgetting the whole data component of all cells, while retaining the graphical aspect. A memory shape is hence a pair composed of a store and a heap shape, $(\mathfrak{s}, \mathfrak{h})$ such that

$-\mathfrak{s}$ is a variable valuation of the form $\mathfrak{s}:$ Var $\rightarrow$ Loc,

$-\mathfrak{h}$ is a partial function $\mathfrak{h}:$ Loc $\rightarrow$ Loc with finite domain.

We will use the typographic convention to differentiate a memory state $(s, h)$ from a memory shape $(\mathfrak{s}, \mathfrak{h})$. Note that concrete stores can be safely identified to abstract stores. We will write shape(.) for the obvious map from concrete heaps to heap shapes:

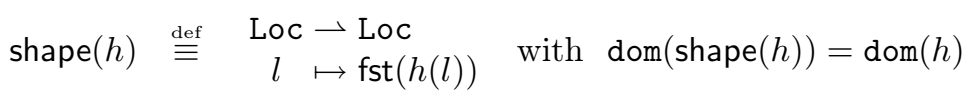

$M S O$ over memory shapes. We assume a set VAR of monadic second-order variables, denoted by $\mathrm{P}, \mathrm{Q}, \mathrm{R}, \ldots$ An environment $\mathcal{E}$ is a map $\mathcal{E}: \mathrm{VAR} \rightarrow \mathcal{P}_{\text {fin }}$ (Loc) that associates to every second order variable a finite set of locations. Since we require finiteness of models, the version of monadic second-order logic we shall consider is usually called weak.

Formulae of (weak) monadic second-order logic (MSO) are defined by the grammar below:

$$
\phi:=\neg \phi|\phi \wedge \phi| \exists x . \phi|x \hookrightarrow y| x=y|\exists \mathrm{P} . \phi| \mathrm{P}(x)
$$


is a valid formula, where Post denotes the strongest postcondition. There are several ways to compute the strongest postcondition of a loop-free sequence of instructions. We sketch here two approaches: the original one in separation logic theory [14], and the one followed by the tool SMALLFoot [1].

The original approach does not make any assumption on the invariant $A$, and fully exploits the magic wand connective. To give an idea, in our case, the postcondition of the loop $P$ of the merge program would look like

$$
\begin{aligned}
& \operatorname{Post}(P, B)=\exists x^{\prime}, y^{\prime}, z^{\prime} . z^{\prime} \hookrightarrow \mathbf{z} \\
& \wedge \operatorname{val}\left(x^{\prime}\right) \geq \operatorname{val}\left(y^{\prime}\right) \Rightarrow\left(x^{\prime} \hookrightarrow \mathbf{x} \wedge y^{\prime}=\mathbf{y}\right. \\
& \left.\wedge\left(\exists v . \quad z^{\prime} \mapsto\left(x^{\prime}, v\right) *\left(z^{\prime} \mapsto(-, v) \rightrightarrows B\left\{x^{\prime}, z^{\prime} / \mathbf{x}, \mathbf{z}\right\}\right)\right)\right) \\
& \wedge \operatorname{val}\left(x^{\prime}\right)<\operatorname{val}\left(y^{\prime}\right) \Rightarrow\left(y^{\prime} \hookrightarrow \mathbf{y} \wedge x^{\prime}=\mathbf{x}\right. \\
& \left.\wedge\left(\exists v . \quad z^{\prime} \mapsto\left(y^{\prime}, v\right) *\left(z^{\prime} \mapsto(-, v) \rightrightarrows B\left\{x^{\prime}, z^{\prime} / \mathbf{x}, \mathbf{z}\right\}\right)\right)\right) .
\end{aligned}
$$

where primed variables quantify over the value of the corresponding program variable before the execution of the loop. What should be underlined concerning this approach is that automatically checking (1) would involve to solve the satisfiability of the logic in presence of magic wand, which is known to be undecidable even with only one selector [6].

In the SMALLFOOT approach, on the contrary, the symbolic computation is not parametric in the invariant. Usually, symbolic memory states are represented by (disjunctions of) formulae of the form $\exists x_{1}, \ldots, x_{n} . \Pi \wedge \Sigma$, where $\Sigma$ (the "spatial" part) is a $*$-conjunct of the elementary list segments present in memory, and $\Pi$ (the "pure" part) handles all other informations that are not properly adressed by local reasoning. For instance, a reasonable loop invariant following this format could be:

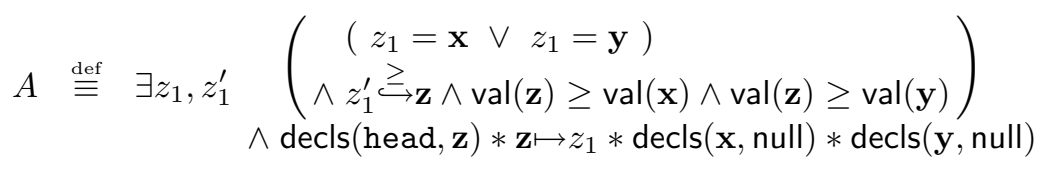

Symbolic computation over lists with values has not been defined in SMALLFoot, but taking inspiration from it, we may consider that for such an invariant the result of the symbolic computation would look like:

$$
\begin{aligned}
& \operatorname{Post}(P, A \wedge \mathbf{x} \neq \operatorname{null} \wedge \mathbf{y} \neq \text { null }) \stackrel{\text { def }}{=} \exists z_{1}^{\prime}, z_{2}, z_{3}, z_{4}, x^{\prime}, y^{\prime}, z^{\prime} . \\
& \left(\begin{array}{c}
\left(\left(z_{2}, z_{3}, z_{4}, y^{\prime}\right)=\left(x^{\prime}, \mathbf{x}, \mathbf{y}, \mathbf{y}\right) \vee\left(z_{2}, z_{3}, z_{4}, x^{\prime}\right)=\left(y^{\prime}, \mathbf{y}, \mathbf{x}, \mathbf{x}\right)\right) \\
\wedge \mathbf{z}=z_{2} \wedge z_{1}^{\prime} \rightleftarrows z^{\prime} \wedge z_{2} \stackrel{\geq}{\leftrightarrows} z_{3} \\
\wedge \operatorname{val}\left(z^{\prime}\right) \geq \operatorname{val}\left(x^{\prime}\right) \wedge \operatorname{val}\left(z^{\prime}\right) \geq \operatorname{val}\left(y^{\prime}\right) \wedge \operatorname{val}\left(z_{2}\right) \geq \operatorname{val}\left(z_{4}\right)
\end{array}\right) \\
& \wedge \operatorname{decls}\left(\text { head, } z^{\prime}\right) * z^{\prime} \mapsto z_{2} * z_{2} \mapsto z_{3} * \operatorname{decls}(\mathbf{x}, \operatorname{null}) * \operatorname{decls}(\mathbf{y}, \text { null })
\end{aligned}
$$

where again primed variables should be thought as the past values of the corresponding program variables. We may underline that, unlike for $(2)$, there are 
long-distance comparisons in the pure part of (3) that involve two quantified variables. As we will see in Section [5, this formula belongs to a fragment for which we obtain an undecidability result. However, looking more carefully to it, one may notice that, out of $z^{\prime}$ and $z_{1}^{\prime}$, all quantified variables are aliased to program variables, which allows to rewrite the formula so that each long-distance comparison involves at least one open variable. Up to that, one may then use our decidability result of Section 5 to automatically check (1).

\section{Decidability of Short-Distance Comparisons}

In this section, we establish the decidability of the short-distance fragment of $\mathrm{SL}_{<}$. This fragment is defined by the following grammar:

$$
\phi::=\neg \phi|\phi \wedge \phi| \exists x . \phi|x \hookrightarrow y| x \stackrel{\leq}{\hookrightarrow} y|x \stackrel{\geq}{\leftrightarrows}| x=y \mid \phi * \phi
$$

(short-distance fragment)

The decidability of satisfiability for this fragment is obtained by reduction to the satisfiability of MSO over shapes.

Colored shapes. We hence have to abstract the values taking care of their local comparisons. To do so, we use a colored shape, with three colors on the edges: 3 : ' $<$ ', ' $>$ ', and '='. In logical terms, these colors will be defined by two second order variables, noted $X$ and $Y$, and we will observe the color ' $=$ ' if both $X$ and $Y$ holds for the source location of the edge, ' $<$ ' if $X$ holds but not $Y$, and ' $>$ ' if $Y$ holds but not $X$. The case where neither $X$ nor $Y$ holds is irrelevant since we assumed a total order on data values, so we should constrain the possible choices for $X$ and $Y$ to avoid this situation. Moreover, some extra constraints will be involved by the necessity to manipulate only colored shapes for which it is possible to assign data respecting the colors (for instance, a cycle of ' $<$ ' cannot be assigned data).

The graph of constraints. Given a shape $(\mathfrak{s}, \mathfrak{h})$, and the interpretations $\mathcal{X}, \mathcal{Y} \subseteq$ $\operatorname{dom}(\mathfrak{h})$ of the second-order variables mentioned before, we define the associated graph of constraints $G=(V, E)$ where:

- $V$ is $\operatorname{dom}(\mathfrak{h})$ quotiented by the equivalence $l \sim l^{\prime}$ relating locations connected by a non oriented, '='-labeled path in the colored shape. Note that each $\sim$-equivalence class contains at most one location $l$ whose image under $\mathfrak{h}$ lies outside the equivalence class of $l$. In such a situation, $[l]$ denotes this equivalence class.

- $E$ is the set of pairs of equivalence classes $\left([l],\left[l^{\prime}\right]\right)$ such that

- either $h(l)=l^{\prime}$ and the color on $l$ is ' $>$ '

- or $h\left(l^{\prime}\right)=l$ and the color on $l^{\prime}$ is ' $<$ '

${ }^{3}$ Formally, on vertices, but each edge can be non-ambiguously identified to its source vertex in a shape. 


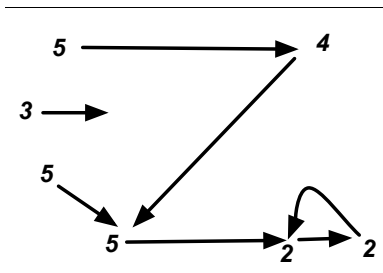

(1)

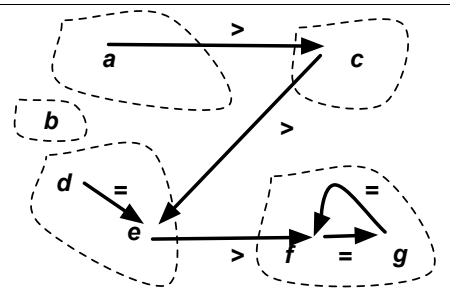

(2)

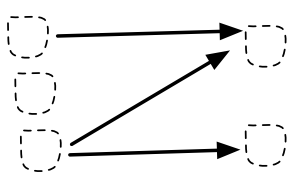

(3)

Fig. 1. A concrete heap (1), its colored abstraction (2), and the associated graph of constraints (3). Here $\mathcal{X}=\{c, d, f, g\}$ and $\mathcal{Y}=\{a, d, e, f, g\}$.

Figure1gives an example of a colored shape and its associated graph of constraints. Note that an edge towards a dangling pointer cannot be colored, and this is in fact the unique situation in which one allows $\neg X \wedge \neg Y$. The graph of constraints helps us to decide whether or not it is possible to assign values to a colored shape: indeed, this problem is equivalent to defining a topological order on the graph of constraints, which is known to be equivalent to this graph being acyclic. What remains to be explained now is: (1) how to define the graph of constraints in MSO, (2) how to express acyclicity, (3) how to treat separating conjunction.

The reduction. The reduction from $\mathrm{SL}_{<}$over memory states to MSO over shapes is defined by $\operatorname{rd}_{\mathrm{SL}_{<} \rightarrow \mathrm{MSO}}(\phi)=\exists X . \exists Y . \exists Z$. Cons $(X, Y, Z) \wedge \operatorname{rd}(\phi, X, Y, Z)$ where:

$-Z$ is an extra second-order variable that is needed to define the current focus, that is the subheap of the original heap on which the (sub)formula is currently evaluated.

- $r d$ is an auxiliary reduction that works assuming that $X, Y$ and $Z$ have been correctly guessed, updating these parameters appropriately when $*$ is translated.

- Cons are constraints imposed on $X, Y$ and $Z$ to guarantee that the first guess is a valid one: $\mathcal{Z}$ is the domain of the heap, and $\mathcal{X}$ and $\mathcal{Y}$ define a colored shape to which one may assign values.

Constraints. We impose three contraints : $\operatorname{Cons}(X, Y, Z) \stackrel{\text { def }}{\equiv} \operatorname{Cons} 1(X, Y, Z) \wedge$ Cons2 $(X, Y, Z) \wedge \operatorname{Cons} 3(X, Y, Z)$

1. the only admitted color on a monochromatic cycle is '=' (this is indeed equivalent to the acyclicity condition on the graph of constraints):

$$
\operatorname{Cons} 1(X, Y, Z) \stackrel{\text { def }}{\equiv} \forall U \subseteq Z . \operatorname{Loop}(U) \Rightarrow(U \subseteq X \Leftrightarrow U \subseteq Y)
$$

where $\operatorname{Loop}(U)$ is defined as $\operatorname{SetOfLoops}(U) \wedge \forall V \subsetneq U \cdot \neg \operatorname{SetOfLoops}(V)$ and $\operatorname{SetOfLoops}(U)$ is $\forall x . U(x) \Rightarrow \exists y . U(y) \wedge y \hookrightarrow x$

2. every edge that should be colored is colored with ' $<$, ' $>$ ' or ' $=$ '

$$
\operatorname{Cons} 2(X, Y, Z) \stackrel{\text { def }}{\equiv} \forall x . \quad(Z(x) \wedge(\exists y . Z(y) \wedge x \hookrightarrow y)) \Leftrightarrow(X(x) \vee Y(x))
$$

3. $\mathcal{Z}$ is the domain of the heap: $\operatorname{Cons} 3(X, Y, Z) \stackrel{\text { def }}{\equiv} \forall x .(x \hookrightarrow-) \Leftrightarrow Z(x)$. 
Let us now state the results we may derive from these definitions. We say that a location $l$ is an increasing (resp. decreasing) node if there are $l^{\prime}, l^{\prime \prime} \in$ Loc and $\alpha, \beta \in$ Dat such that $h(l)=\left(l^{\prime}, \alpha\right), h\left(l^{\prime}\right)=\left(l^{\prime \prime}, \beta\right)$, and $\alpha \leq \beta$ (resp. $\left.\alpha \geq \beta\right)$. We write $\operatorname{dom}^{+}(h)$ (resp. $\left.\operatorname{dom}^{-}(h)\right)$ to denote the set of increasing (resp. decreasing) nodes of $h$, and $\mathcal{E}_{h}$ denotes $\left[X \mapsto \operatorname{dom}^{+}(h), Y \mapsto \operatorname{dom}^{-}(h), Z \mapsto \operatorname{dom}(h)\right]$.

Lemma 1 (Constraints soundness). If $(\mathfrak{s}, \mathfrak{h}), \mathcal{E} \models_{\text {MSO }} \operatorname{Cons}(X, Y, Z)$ then there is a $h:$ Loc $\rightarrow$ Loc $\times$ Dat such that shape $(h)=\mathfrak{h}, \mathcal{E}(Z)=\operatorname{dom}(h)$, $\mathcal{E}(X)=\operatorname{dom}^{+}(h)$ and $\mathcal{E}(Y)=\operatorname{dom}^{-}(h)$.

Lemma 2 (Constraints completeness). For all models with data $(s, h)$ :

$$
(s, \operatorname{shape}(h)), \mathcal{E}_{h} \models_{\text {MSO }} \operatorname{Cons}(X, Y, Z) .
$$

Auxiliary recursive translation. The auxiliary recursive translation rd is defined as follows: (1) it is isomorphic on the cases of $\phi \wedge \psi, \neg \phi, \exists x . \phi$, and $x=y$, and (2) for other connectives, parameters $X, Y, Z$ come into play:

$$
\begin{aligned}
& \operatorname{rd}(x \hookrightarrow y, X, Y, Z) \stackrel{\text { def }}{=} Z(x) \wedge x \hookrightarrow y \\
& \operatorname{rd}(x \stackrel{\leq}{\leftrightarrows} y, X, Y, Z) \quad \stackrel{\text { def }}{=} Z(x) \wedge Z(y) \wedge X(x) \wedge x \hookrightarrow y \\
& \operatorname{rd}(x \stackrel{\geq}{\hookrightarrow} y, X, Y, Z) \stackrel{\text { def }}{=} Z(x) \wedge Z(y) \wedge Y(x) \wedge x \hookrightarrow y \\
& \operatorname{rd}\left(\phi_{1} * \phi_{2}, X, Y, Z\right) \stackrel{\text { def }}{\equiv} \exists Z_{1}, Z_{2} \text {. } \\
& \operatorname{rd}\left(\phi_{1}, X, Y, Z_{1}\right) \wedge \operatorname{rd}\left(\phi_{2}, X, Y, Z_{2}\right) \wedge Z=Z_{1} \cup Z_{2} \wedge Z_{1} \cap Z_{2}=\emptyset
\end{aligned}
$$

Lemma 3 (Reduction Lemma). For all $s, h$, for all $\mathcal{Z} \subseteq \operatorname{dom}(h)$, $(s, \operatorname{shape}(h)), \mathcal{E}_{h}[Z \mapsto \mathcal{Z}] \models_{\text {MSO }} \operatorname{rd}(\phi, X, Y, Z)$ if and only if $\left(s, h_{\mid \mathcal{Z}}\right) \models_{\mathrm{SL}} \phi$.

Theorem 2. For all formulae $\phi$ of $\mathrm{SL}_{<}$, there exists $(s, h)$ such that $(s, h) \models_{\mathrm{SL}} \phi$

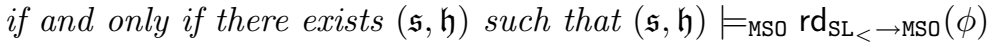

Thanks to Theorem 2 and Theorem 1, we have established the announced result:

Corollary 1. The satisfiability problem for the fragment of $\mathrm{SL}_{<}$with shortdistance comparisons is decidable.

\section{Long-Distance Comparisons}

\subsection{An Undecidablity Result}

We consider now the fragment of $\mathrm{SL}_{<}$where magic wand is still dropped, but long-distance comparison is considered:

$\phi::=\neg \phi|\phi \wedge \phi| \exists x . \phi|\exists v . \phi| x \hookrightarrow y|\operatorname{val}(x) \leq v| \operatorname{val}(x) \geq v|x=y| \phi * \phi$.

(long-distance fragment)

We show that, without any further restriction, long-distance comparisons yield undecidability, even for a simpler fragment:

$\phi::=\neg \phi|\phi \wedge \phi| \exists x . \phi|x \hookrightarrow y| \operatorname{val}(x)=\operatorname{val}(y)|x=y| \phi * \phi$.

(equality long-distance fragment) 
Theorem 3. The satisfiability problem for the equality long-distance fragment is undecidable.

The proof goes by reduction to the satisfiability problem of first-order formulae over data words. Before giving the intuition of the reduction, we first recall this logic.

First-order logic over data words. We assume a finite set $\Sigma$. A finite data word is a sequence $w=w_{1} . . w_{n}$, where $w_{i}=\left(a_{i}, \alpha_{i}\right) \in \Sigma \times$ Dat; we write $|w|$ to denote the length $n \in \mathbb{N}$ of $w$. Note that, so far, we assumed a total order on Dat, but this aspect is not essential for this reduction, and one may think of Dat as any arbitrary infinite set. The first-order formulae we will evaluate over these models are defined by the following grammar:

$$
\text { (FO over data words) } \quad \phi::=\neg \phi|\phi \wedge \phi| \exists x . \phi|a(x)| x=y+1 \mid x \sim_{\text {Dat }} y
$$

where $a \in \Sigma$. Variables are interpreted as positions in the word through a valuation $\sigma: \operatorname{Var} \rightarrow\{1 . .|w|\},+1$ is the standard addition over $\mathbb{N}$, and $\sim_{\text {Dat }}$ relates positions holding the same datum. More formally

$$
\begin{aligned}
& w, \sigma \models_{F O} \exists x . \phi \text { if there is } n \in\{1 . .|w|\} \text { s.t. } w, \sigma[x \mapsto n] \models_{F O} \phi \\
& w, \sigma \models_{F O} a(x) \text { if } a_{\sigma(x)}=a \\
& w, \sigma \models_{F O} x=y+1 \text { if } \sigma(x)=\sigma(y)+1 \\
& w, \sigma \models_{F O} x \sim_{\text {Dat }} y \text { if } \alpha_{\sigma(x)}=\alpha_{\sigma(y)}
\end{aligned}
$$

Theorem 4 (see [2], Prop. 27). The satisfiability problem for a closed sentence of FO over data words is undecidable.

The reduction. To prove Theorem 3 , we define a translation from FO to the long-distance fragment such that a formula $\phi$ admits a data word model if and only if its translation admits a memory state model. A data word of length $n$ is encoded as a list segment of length $2 n$, placing the sequence of letters of $\Sigma$ in the even positions, and the data sequence in odd positions. Then $x=y+1$ can be encoded by $y \hookrightarrow^{2} x$, and $x \sim_{\text {Dat }} y$ can be encoded by $\operatorname{val}(x)=\operatorname{val}(y)$.

\subsection{Decidability of Guarded Long-Distance Comparisons}

We now consider the fragment of formulae where every quantification over values is restricted to values stored in the cells that are pointed by the program variables:

$$
\begin{aligned}
\phi::= & \rightarrow \phi|\phi \wedge \phi| \exists x \cdot \phi \mid \exists v \cdot \operatorname{val}(\mathbf{x})=v \wedge \phi \\
& |x \stackrel{\leq}{\leftrightarrows} y| x \stackrel{\geq}{\hookrightarrow} y|x \hookrightarrow y| \operatorname{val}(x) \leq v|\operatorname{val}(x) \geq v| x=y \mid \phi * \phi .
\end{aligned}
$$

(guarded long-distance fragment)

Note that guarded long-distance comparisons are quite weak, and we need to add short-distance comparisons as basic predicates if we still want to use them.

Theorem 5. The satisfiability problem for the guarded long-distance fragment is decidable. 
Proof of Theorem 5. We only sketch the proof. We adapt the proof of Theorem 2 by extending the notions of colored shapes and graphs of constraints. Let ProgVar $=\left\{\mathbf{x}_{1}, . ., \mathbf{x}_{n}\right\} \subsetneq$ Var be a finite set of variables such that every formula to be translated will have all its open variables in ProgVar. To every variable $\mathbf{x} \in$ ProgVar, we associate two second-order variable $X_{\mathbf{x}}, Y_{\mathbf{x}}$. A colored shape is then a tuple

$$
C S=\left((\mathfrak{s}, \mathfrak{h}), \mathcal{X}, \mathcal{Y}, \mathcal{X}_{\mathbf{x}_{1}}, \mathcal{Y}_{\mathbf{x}_{1}}, \ldots, \mathcal{X}_{\mathbf{x}_{n}}, \mathcal{Y}_{\mathbf{x}_{n}}\right)
$$

where $\mathcal{X}_{\mathbf{x}}, \mathcal{Y}_{\mathbf{x}}$ are finite sets of locations; it is well defined if $\mathcal{X} \cup \mathcal{Y}=\operatorname{dom}(h) \cap$ $h^{-1}(\operatorname{dom}(h))$ and $\mathcal{X}_{\mathbf{x}} \cup \mathcal{Y}_{\mathbf{x}}=\operatorname{dom}(h)$ for every program variable $\mathbf{x}$ such that $\mathfrak{s}(\mathbf{x}) \in \operatorname{dom}(h)$. Let $(\mathfrak{s}, \mathfrak{h})$ be a fixed shape. We define the relation $\sim$ on $\operatorname{dom}(\mathfrak{h})$ as the smallest equivalence relation such that:

- if $l \in \mathcal{X}_{\mathbf{x}} \cap \mathcal{Y}_{\mathbf{x}}$ and $\mathfrak{s}(\mathbf{x}) \in \operatorname{dom}(\mathfrak{h})$, then $\mathfrak{s}(\mathbf{x}) \sim l$;

- if $\mathfrak{h}(l)=l^{\prime}$, and $l \in \mathcal{X} \cap \mathcal{Y}$, then $l \sim l^{\prime}$.

The graph of constraints associated to $C S$ is the pair $(V, E)$ where the vertex set $V$ is the quotient of $\operatorname{dom}(\mathfrak{h})$ by $\sim$, and there is an edge from the equivalence class $c_{1}$ to $c_{2}$ if at least one of the following conditions holds:

- either there is $\mathfrak{s}(\mathbf{x}) \in c_{1}$ and $l \in c_{2}$ such that $l \in \mathcal{Y}_{\mathbf{x}}-\mathcal{X}_{\mathbf{x}}$;

- or there is $\mathfrak{s}(\mathbf{x}) \in c_{2}$ and $l \in c_{1}$ such that $l \in \mathcal{X}_{\mathbf{x}}-\mathcal{Y}_{\mathbf{x}}$;

- or there is $l \in c_{1}, l^{\prime} \in c_{2}$ such that $\mathfrak{h}(l)=l^{\prime}$ and $l \in \mathcal{Y}-\mathcal{X}$;

- or there is $l \in c_{1}, l^{\prime} \in c_{2}$ such that $\mathfrak{h}\left(l^{\prime}\right)=l$ and $l^{\prime} \in \mathcal{X}-\mathcal{Y}$.

It is possible to check that the graph of constraints and the acyclicity condition on it are MSO definable. We may then adapt the reduction of Section 4 , we guess the $\mathcal{X}_{\mathrm{x}} \mathrm{s}$ and $\mathcal{Y}_{\mathrm{x}} \mathrm{s}$ at start and check we made a valid guess, and we extend the recursive translation $\mathrm{rd}(\phi)$ with the following cases:

$$
\begin{array}{ll}
\operatorname{rd}(\exists v \cdot \operatorname{val}(\mathbf{x})=v \wedge \phi) & \stackrel{\text { def }}{\equiv} \operatorname{rd}(\phi\{\operatorname{val}(\mathbf{x}) / v\}) \\
\operatorname{rd}(\operatorname{val}(x) \leq \operatorname{val}(\mathbf{x})) & \stackrel{\text { def }}{\equiv} Z(x) \wedge Z(\mathbf{x}) \wedge Y_{\mathbf{x}}(x) \wedge \neg X_{\mathbf{x}}(x) \\
\operatorname{rd}(\operatorname{val}(x) \geq \operatorname{val}(\mathbf{x})) & \stackrel{\text { def }}{\equiv} Z(x) \wedge Z(\mathbf{x}) \wedge X_{\mathbf{x}}(x) \wedge \neg Y_{\mathbf{x}}(x)
\end{array}
$$

Perspectives. We expect this decidability result to extend to more complex data structures that would have a decidable MSO theory (trees, doubly-linked lists, lists of lists, and more generally tree-width bounded structures), and to more complex short-distance comparisons ( $n$-th successor, brothers,...). Moreover, such restrictions may be sufficient to handle other interesting applications, for instance search-trees. In this sense, we claim that the graph of constraints is the "right" general concept for logics dealing with sorted data structures.

\section{Magic Wand and Restricted Magic Wand}

Even without data, the logic with the operator $*$ is proved to be undecidable in [6]. In the technical report [5] corresponding to the paper, a decidable 
separation logic with a restricted magic wand is presented. Let us write the definition of this binary operator, $*_{n}$ (for $n$ an integer). Unlike the plain operator $*$, the quantification on disjoint heaps of $*_{n}$ considers only heaps for which the cardinality of the domain is bounded by $n$. More formally, we define that $(\mathfrak{s}, \mathfrak{h}) \models \phi_{1} *_{n} \phi_{2}$ if and only if for all $\mathfrak{h}^{\prime}$ such that $\mathfrak{h}^{\prime} \perp \mathfrak{h}$ and $\left|\operatorname{dom}\left(\mathfrak{h}^{\prime}\right)\right| \leq n$, if $\left(\mathfrak{s}, \mathfrak{h}^{\prime}\right) \models \phi_{1}$ then $\left(\mathfrak{s}, \mathfrak{h} * \mathfrak{h}^{\prime}\right) \models \phi_{2}$. It can be seen as an abbreviation of $\left(\phi_{1} \wedge \neg \exists x_{1}, \ldots, x_{n+1} \cdot \bigwedge_{i \neq j} x_{i} \neq x_{j} \wedge \bigwedge_{i} \exists y \cdot x_{i} \hookrightarrow y\right) * \phi_{2}$. In the sequel, we will prove that, in the context of heaps with data, $*_{1}$ is sufficient to obtain undecidability.

Let $R$ denote an arbitrary binary relation on Dat. Let us call $\sim_{R}$ the equivalence relation defined as $\alpha \sim_{R} \alpha^{\prime}$ iff $\{\beta, \beta R R \alpha\}=\left\{\beta, \beta R R \alpha^{\prime}\right\}$. We consider the atomic formula $\operatorname{val}(x) R \operatorname{val}(y)$ stating that values stored in $x$ and $y$ compare through $R$. Formally, $(s, h) \models_{\mathrm{SL}} \operatorname{val}(x) R \operatorname{val}(y)$ iff there are $\alpha, \beta \in \mathrm{Val}$ and $l, l^{\prime} \in$ Loc such that $h(s(x))=(l, \alpha), h(s(y))=\left(l^{\prime}, \beta\right)$, and $\alpha R \beta$.

We now introduce the relation $x \stackrel{R}{\hookrightarrow} y$ for $x \hookrightarrow y \wedge \operatorname{val}(x) R \operatorname{val}(y)$, and define the $\operatorname{logic} \operatorname{SL}\left(R, *_{1}\right)$ with the grammar:

$$
\phi::=\neg \phi|\phi \wedge \phi| \exists x . \phi|x \hookrightarrow y| x \stackrel{R}{\hookrightarrow} y|x=y| \phi * \phi \mid \phi-*_{1} \phi .
$$

We are going to prove that satisfiability and validity problems are undecidable for $\operatorname{SL}\left(R, *_{1}\right)$, for any $R \in\{\leq, \geq,=,<,>\}$. We will rely on the previous section, especially Theorem $[$ by simulating a long-distance equality. We first need the following fact:

Lemma 4. Let $R \in\{\leq, \geq,=,<,>\}$. Then $\sim_{R}$ has an infinite number of equivalence classes.

Let $\sim$ be an equivalence relation on Dat with infinitely many equivalence classes. Let us define the following fragment:

$$
\phi::=\neg \phi|\phi \wedge \phi| \exists x . \phi|x \hookrightarrow y| \operatorname{val}(x) \sim \operatorname{val}(y)|x=y| \phi * \phi .
$$

(equivalence long-distance fragment)

Then the following lemma, a slight variation of Theorem 3 also holds in this generalised framework:

Lemma 5. The satisfiability problem for the equivalence long-distance fragment is undecidable.

Proof. By the same encoding as the one of Theorem 3, one may reduce a satisfiability problem of an FO sentence over data words, where data taken from the infinite quotient set Dat $/ \sim_{R}$, to the satisfiability problem for the equivalence long-distance fragment.

Lemma 6. There is a formula $\phi_{R}\left(x, x^{\prime}\right) \in \mathrm{SL}\left(R, *_{1}\right)$ such that for all $(s, h)$ with $\left\{s(x), s\left(x^{\prime}\right)\right\} \subseteq \operatorname{dom}(h)$ :

$$
(s, h) \models \phi_{R}\left(x, x^{\prime}\right) \text { iff }(s, h) \models \operatorname{val}(x) \sim_{R} \operatorname{val}\left(x^{\prime}\right)
$$


We only sketch the proof. $\phi \Re_{1} \psi$ will abbreviate $\neg\left(\phi *_{1} \neg \psi\right)$. Then $(s, h) \models \phi \Re_{1} \psi$ iff there is $h^{\prime}$ such that $\left(s, h^{\prime}\right) \models \phi,\left(s, h * h^{\prime}\right) \models \psi$ and $\left|\operatorname{dom}\left(\mathfrak{h}^{\prime}\right)\right| \leq 1$. The operators $*_{1}$ and $\nVdash_{1}$ will be used to simulate restricted quantifications over Dat, respectively universal and existential. Consider the formula $\phi$

$$
\begin{gathered}
\exists x_{1} \cdot \exists x_{2} \cdot\left(\neg \exists x_{3} \cdot x_{1} \hookrightarrow x_{3} \vee x_{2} \hookrightarrow x_{3}\right) \\
\wedge\left(x_{1} \hookrightarrow x_{2}\right) *_{1}\left(\left(\operatorname{val}\left(x_{1}\right) R R \operatorname{val}(x)\right) \Leftrightarrow \quad\left(\operatorname{val}\left(x_{1}\right) R R \operatorname{val}\left(x^{\prime}\right)\right)\right)
\end{gathered}
$$

where $\operatorname{val}\left(x_{1}\right) R R \operatorname{val}(x)$ abbreviates $\left(x_{2} \hookrightarrow x\right) \Im_{1}\left[\left(x_{1} \stackrel{R}{\hookrightarrow} x_{2}\right) \wedge x_{2} \stackrel{R}{\hookrightarrow} x\right]$. The formula $\phi$ expresses that for all $\alpha$, there is $\beta$ such that $\alpha R \beta R \operatorname{snd}(h(s(x)))$ if and only if there is $\beta$ such that $\alpha R \beta R$ snd $\left(h\left(s\left(x^{\prime}\right)\right)\right)$, that is $\operatorname{val}(x) \sim_{R} \operatorname{val}\left(x^{\prime}\right)$. As a consequence:

Theorem 6. For any $R \in\{\leq, \geq,<,>,=\}$, the validity and satisfiability problems for $\operatorname{SL}\left(R, *_{1}\right)$ are undecidable.

\section{Conclusion}

Our results give a wide picture of the decidability status of the satisfiability problem for separation logic dealing with data.

With the ability to describe lists and quantify over locations, allowing longdistance comparisons brings undecidability, and so does allowing the operator $*$, even strongly restricted. Yet, there is a very positive result: dropping these two features makes the satisfiability problem decidable, still being able to do local reasoning and express properties about ordered recursive structures. The decidability even holds when a finite set of references can be compared to all the rest of the memory.

Some ways to restrict the full language are still unexplored, for instance bounding the amount of quantified variables. With the same hope to obtain decidability for satisfiability problems, one may look at extension of our decidable fragment. For instance, our results are general for any totally ordered infinite set, and questions remain open about partially ordered sets.

\section{References}

1. Berdine, J., Calcagno, C., O'Hearn, P.: Smallfoot: Modular automatic assertion checking with separation logic. In: de Boer, F.S., Bonsangue, M.M., Graf, S., de Roever, W.-P. (eds.) FMCO 2005. LNCS, vol. 4111, pp. 115-137. Springer, Heidelberg (2006)

2. Bojanczyk, M., Muscholl, A., Schwentick, T., Segoufin, L., David, C.: Two-variable logic on words with data. In: Proceedings of 21th IEEE Symposium on Logic in Computer Science (LICS 2006), 12-15 August 2006, Seattle, WA, USA, pp. 7-16 (2006)

3. Bouajjani, A., Bozga, M., Habermehl, P., Iosif, R., Moro, P., Vojnar, T.: Programs with lists are counter automata. In: Ball, T., Jones, R.B. (eds.) CAV 2006. LNCS, vol. 4144, pp. 517-531. Springer, Heidelberg (2006) 
4. Bozga, M., Iosif, R., Lakhnech, Y.: On logics of aliasing. In: Giacobazzi, R. (ed.) SAS 2004. LNCS, vol. 3148, pp. 344-360. Springer, Heidelberg (2004)

5. Brochenin, R., Demri, S., Lozes, E.: On the almighty wand. Technical report, LSV, ENS de Cachan (2008)

6. Brochenin, R., Demri, S., Lozes, É.: On the almighty wand (To appear). In: Kaminski, M., Martini, S. (eds.) CSL 2008. LNCS, vol. 5213, pp. 323-338. Springer, Heidelberg (2008)

7. Calcagno, C., Yang, H., O'Hearn, P.: Computability and complexity results for a spatial assertion language for data structures. In: Hariharan, R., Mukund, M., Vinay, V. (eds.) FSTTCS 2001. LNCS, vol. 2245, pp. 108-119. Springer, Heidelberg (2001)

8. Demri, S., Lazić, R., Nowak, D.: On the freeze quantifier in constraint LTL: Decidability and complexity. In: Proceedings of the 12th International Symposium on Temporal Representation and Reasoning (TIME 2005), Burlington, Vermont, USA, pp. 113-121. IEEE Computer Society Press, Los Alamitos (2005)

9. Jensen, J., Jorgensen, M., Klarlund, N., Schwartzbach, M.: Automatic verification of pointer programs using monadic second-order logic. In: PLDI 1997, pp. 226-236. ACM, New York (1997)

10. Lev-Ami, T., Sagiv, M.: TVLA: A system for implementing static analyses. In: Palsberg, J. (ed.) SAS 2000. LNCS, vol. 1824, pp. 280-302. Springer, Heidelberg (2000)

11. Loginov, A., Reps, T., Sagiv, M.: Refinement-based verification for possibly-cyclic lists. In: Reps, T., Sagiv, M., Bauer, J. (eds.) Wilhelm Festschrift. LNCS, vol. 4444, pp. 247-272. Springer, Heidelberg (2007)

12. Nguyen, H.H., David, C., Qin, S.C., Chin, W.-N.: Automated verification of shape and size properties via separation logic. In: Cook, B., Podelski, A. (eds.) VMCAI 2007. LNCS, vol. 4349, pp. 251-266. Springer, Heidelberg (2007)

13. Rabin, M.: Decidability of second-order theories and automata on infinite trees. Transactions of the American Mathematical Society 41, 1-35 (1969)

14. Reynolds, J.C.: Separation logic: a logic for shared mutable data structures. In: LICS 2002, pp. 55-74. IEEE, Los Alamitos (2002)

15. Trakhtenbrot, B.A.: The impossibility of an algorithm for the decision problem for finite models. Dokl. Akad. Nauk SSSR 70, 572-596 (1950); English translation in: AMS Transl. Ser. 2, 23(1063), 1-6

16. Yorsh, G., Rabinovich, A.M., Sagiv, M., Meyer, A., Bouajjani, A.: A logic of reachable patterns in linked data structures. In: Sassone, V. (ed.) FOSSACS 2005. LNCS, vol. 3441, pp. 94-110. Springer, Heidelberg (2005) 